\title{
El abordaje de la Atención Primaria de Salud, modelos organizativos y prácticas: caso de un Centro de Salud público urbano de Montevideo, Uruguay 2011
}

The approach of Primary Health Care, organizative models and practices: case of a urban public Health Center in Montevideo, Uruguay 2011

Rosario Berterretche; Ana Sollazzo $^{2}$

${ }^{1}$ Especialista en Administración de Servicios de Salud. Professora Agda. Unidad Docente de Administración de Servicios de Salud, Universidad de la República - Montevideo, Uruguay. anasollazzo@gmail.com

${ }^{2}$ Professora Adjunta do Departamento de Medicina Preventiva y Social, Facultad de Medicina. Universidad de la República - Montevideo, Uruguay. rosariobe@gmail.com
RESUMEN El estudio de caso tuvo lugar en un Centro de Salud público de Montevideo, con el objetivo de comprender cómo los modelos organizativos de oferta y gestión de la APS son traducidos en servicios, prácticas y procesos asistenciales. El Instrumento de investigación utilizado se contextualizó en la herramienta creada por la Profa. B. Starfield, el Primary Care Assessment Tool, adaptada y validada por Almeida y Macinko en Brasilia en 2006. Se aplicaron los cuestionarios a un grupo de usuarios, profesionales y gestores seleccionados a través de un muestreo no probabilístico por criterios. Desde un punto de vista general, se observa una buena calificación de desempeño a nivel global en los atributos: puerta de entrada, cartera de servicios y formación profesional. Los atributos de nivel intermedio de desempeño corresponden a vínculo y coordinación. En el otro extremo, los atributos con menor nivel de desempeño fueron el enfoque familiar y comunitario y el acceso. Si bien la estrategia de APS está consagrada a un nivel macro social a través de un marco normativo sólido, su implementación a nivel operativo implica importantes desafíos para el logro de una adecuada integración y coordinación de los cuidados.

PALABRAS CLAVE: Atención Primaria de Salud, atributos APS, organización de servicios de salud.

ABSTRACT The study case took place at a public health center of Montevideo with the aim of understanding how the organizational models of provision and management of PHC services are translated into practices and care processes. The research instrument used was contextualized in the tool created by Prof.. B. Starfield, the Primary Care Assessment Tool, adapted and validated by Almeida and Macinko in Brasilia in 2006. Questionnaires were applied to a group of users, professionals and managers selected through a non-probabilistic sampling criteria. From a general point of view, there is a good performance rating globally in the attributes: gateway, service portfolio and profesional training. Intermediate attributes correspond to link performance and coordination. At the other extreme, the attributes with lower performance were the family and community focus and access. While the PHC strategy is devoted to social macro level through a strong regulatory framework, its implementation at operational level involves major challenges for achieving proper integration and coordination of care.

KEYWORDS: Primary Health Care; PHC Atributes; Health Services Organization. 


\section{Introducción}

La segmentación y la fragmentación de los sistemas de salud constituyen las categorías de análisis centrales del estudio multicéntrico en Atención Primaria de Salud (APS), modelos asistenciales, integración al sistema de salud e intersectorialidad en contextos urbanos en Argentina, Brasil Paraguay y Uruguay.

Existen diferentes enfoques con respecto a la APS y a su implementación. El enfoque de la APS renovada expresada por la Organización Panamericana de la Salud (OPS) en la Declaración de Montevideo (OPS, 2007) plantea una concepción abarcadora, entendiendo la APS como una política de reorganización del modelo asistencial y una estrategia para el fortalecimiento del sistema de salud como un todo.

En este sentido, la OPS ha identificado barreras y factores facilitadores para la puesta en marcha exitosa de la APS en la región de las Américas (MACINKO et $a l, 2007)$. Entre los factores percibidos como barreras se describen además de la segmentación, la falta de compromiso político, la poca coordinación entre la comunidad y los organismos competentes, el uso inadecuado de la información, la insuficiente colaboración intersectorial y la insuficiente inversión en recursos humanos. Se plantea además que el desarrollo de sistemas de salud basados en APS impone grandes desafíos para los sistemas de recursos humanos. (MACINKO et al, 2007).

Entre los factores que se describen como facilitadores para la implementación de la APS desde una concepción abarcadora y a nivel macrosocial se identifican la multifactorialidad de los determinantes de la salud, el compromiso político, el adecuado financiamiento y la creación de un marco institucional y legal que coadyuve a mejorar la calidad. A nivel de la prestación de los servicios, se identifican su orientación hacia la promoción de la salud y la prevención de la enfermedad, la integración de los servicios, el enfoque hacia la familia y la comunidad (MACINKO et al, 2007).

El modelo analítico propuesto en la investigación consideró el contexto macrosocial en que se desarrolla el sistema de salud, las condiciones particulares del sector $\mathrm{y}$, por último, el nivel operativo en el que se desarrolla la APS. A nivel macrosocial se identifican las condiciones del contexto social, político y económico de los países, así como las condiciones de vida, demográficas y epidemiológicas de la población. En cuanto a las condiciones sectoriales, se identifican la conducción y el financiamiento del sistema, los recursos abocados al sector, así como los arreglos organizacionales que hacen a la integralidad y la continuidad del proceso de atención y la articulación intersectorial. Finalmente, en el nivel operativo es donde se expresan los atributos de la APS y se observa su desempeño en relación al acceso de los servicios y su función de primer contacto, la existencia de una cartera integral de servicios coordinados y con enfoque familiar, orientados a la comunidad y con recursos humanos formados en el enfoque de APS (CASSADY et al., 2000).

La primera fase de esta investigación consistió en un estudio panorámico realizado en 2009 en Uruguay. En esta fase se analizaron los dos primeros aspectos a los que hace referencia el modelo analítico: los factores del contexto macro social y los elementos del sistema de salud que condicionan el desempeño de la APS (SOLLAZZO; BERTERRETCHE, 2011).

Este artículo presenta los resultados de la segunda fase en Uruguay del estudio multicéntrico en APS. En esta fase se desarrollaron estudios de caso en centros urbanos de Uruguay, Paraguay y Argentina. Los grandes centros urbanos se caracterizan por una elevada densidad poblacional, contexto en el que se expresan fuerzas sociales con distintos proyectos e intereses en el sector salud, así como la mayor concentración de prestadores de servicios con diferentes grados de complejidad tecnológica diagnóstica y terapéutica, así como la mayor posibilidad de acceso de la población. En los grandes centros urbanos, la segmentación y fragmentación del sistema están presentes en toda su complejidad, lo que posibilita la identificación de los principales problemas y de factores que facilitan y limitan la implementación de una APS abarcadora y de la integración vertical y horizontal.

El estudio de caso aborda en el nivel operativo los atributos de la APS presentes en las prestaciones de los servicios de salud a través de la percepción de los diferentes actores involucrados: gestores, profesionales y usuarios. 
En Uruguay, el estudio de caso fue realizado en el Centro de Salud Unión de la Administración de Servicios de Salud del Estado (ASSE) durante 2011. ASSE es el principal prestador público de servicios de salud del país que contaba con 1.205.026 en Setiembre de 2011 (ASSE, 2011).

Asiste a los segmentos más frágiles de la población, en tanto congrega en sus usuarios a las personas con mayor vulnerabilidad social en todos los grupos etarios. Cuenta con una red de más de 700 centros que prestan servicios, dentro de los que se destacan Hospitales especializados en el área de la niñez, el adulto mayor, las patologías psiquiátricas y de drogodependencia, el cáncer, la oftalmología, la traumatología y la reumatología, incluyendo también la mayor red de atención del primer nivel con Centros de Salud y Policlínicas en todo el territorio nacional (ASSE, 2011).

\section{Su misión es}

ser el prestador público de referencia, basado en la Atención Primaria, con equidad, eficiencia y calidad, y con capacidad para responder a las necesidades de su población usuaria, en un marco de políticas de equidad social. (ASSE, 2010).

En este marco se ha definido como principal lineamiento estratégico avanzar en el cambio del modelo fortaleciendo el primer nivel de atención para aumentar su capacidad de resolución en el marco de la estrategia de Atención Primaria de Salud (ASSE, 2011).

Es así que en el transcurso de 2010 se conforma el Primer Nivel de Atención (PNA) a nivel Nacional. En este sentido, se crean 19 Unidades Ejecutoras de PNA constituyéndose Redes Departamentales de PNA. Estos servicios se interrelacionan con los otros niveles de atención conformando una red nacional de servicios (ASSE, 2011).

La Red de Atención del Primer Nivel (RAP) del Área Metropolitana integra 114 puntos de contacto con la población y brinda servicios a 350.000 usuarios de Montevideo y zona metropolitana. Desde 2005 se ha procesado una reorganización de base territorial, definiéndose tres regiones de salud que contienen seis zonas de salud, totalizando 18 zonas en el departamento de Montevideo (BENIA et al., 2011).
El Centro de Salud Unión forma parte de la zona este de la RAP contando con una oferta de especialidades básicas y especialistas de referencia para la zona este de Montevideo, brindando atención integral a niños y adultos beneficiarios de ASSE de la zona de referencia. El Centro de Salud Unión tiene a su cargo dos policlínicas dependientes, León Duarte y Maroñas, y seis consultorios de Medicina Familiar. Cuenta con 95 funcionarios y algunos servicios contratados (CRUZ, 2011). La población usuaria del área de responsabilidad se estima en 23.000 habitantes. El área de referencia abarca más de 500 manzanas (cada manzana de zona urbana tiene una superficie de una hectárea) en los barrios de mayor urbanización y se estiman en unas 50 manzanas más los espacios de asentamientos irregulares (zonas no urbanizadas) (CRUZ, 2011).

Mensualmente se realizan aproximadamente 5.000 consultas (lo que incluye el Centro de Salud y sus ocho policlínicas periféricas). Solo en el Centro de Salud se realizan 3.200 consultas por mes; correspondiendo a pacientes adultos que se asisten en Medicina General, 500 consultas y 480 consultas a niños que concurren a Pediatría; el resto de las consultas son de las diferentes especialidades médicas. En las policlínicas periféricas se realizan aproximadamente 1.000 consultas mensuales de pacientes adultos y 800 consultas mensuales de niños (LEVCOVITZ et al, 2011; CRUZ, 2011).

El objetivo del presente estudio de caso fue comprender cómo los abordajes de APS y los correspondientes modelos organizacionales de oferta y gestión de la APS son traducidos en servicios, prácticas y procesos asistenciales.

\section{Metodología}

El estudio de caso se realizó en el Centro de Salud Unión y sus policlínicas y consultorios dependientes, perteneciente a la RAP. Esta selección se hizo de forma consensuada con los 'decisores'.

El Instrumento de investigación utilizado se contextualizó en la herramienta creada por la Profa ${ }^{a}$ B. Starfield, el Primary Care Assessment Tool (PCAT), desarrollada por la John Hopkins Primary Care Policy Centre for Underserved Populations. Dicha 
herramienta fue adaptada y validada por Celia Almeida y James Macinko en Brasilia en el ańo 2006. El equipo de investigación del estudio multicéntrico realizó un encuentro metodológico, acordando por consenso entre los países participantes la utilización de tres formularios semiestructurados dirigidos a usuarios, profesionales y gestores de los servicios de Salud de Primer Nivel de Atención de las versiones validadas por Almeida y Macinko (2006) previa autorización de los autores. Cada instrumento contiene preguntas cerradas que valoran los atributos de la APS. Las preguntas que exploran el desempeño de los atributos de la APS poseen categorías de respuestas cerradas que incluyen una escala valorativa preestablecida.

Los atributos de la APS que explora el instrumento utilizado son (VEGA ROMERO; MARTÍNEZ COLLANTES; ACOSTA RAMIREZ, 2009):

- Acceso: oportunidad de utilización de los servicios por la ausencia de barreras económicas, geográficas, organizacionales y culturales

- Puerta de entrada (primer contacto): existencia de una fuente regular de atención disponible dirigida a la atención en salud de la población.

- Vínculo (longitudinalidad): posibilidad de lograr una atención en salud permanente en el tiempo, así como regularidad en la relación entre el usuario y un proveedor estable de los servicios médicos.

- Cartera integral de servicios (integralidad): conjunto de servicios disponibles que permitan cubrir las necesidades y problemáticas de salud de los diversos grupos que conforman la población usuaria. Incluye los diversos componentes de la atención en salud: promoción, prevención, curación y rehabilitación.

- Coordinación: existencia de mecanismos de comunicación, referencia y contrareferencia entre los diversos proveedores de servicios, para evitar la fragmentación de la atención en salud.
- Enfoque familiar: considera la importancia del entorno y los antecedentes familiares dentro de los condicionantes del proceso saludenfermedad, así como la necesidad de facilitar y fomentar la participación y apoyo familiar en la atención en salud.

- Orientación a la comunidad: fomento de la participación comunitaria en la definición de necesidades y en las alternativas para su intervención.

- Formación profesional: recursos humanos con capacitación y formación en el enfoque de APS para facilitar el cumplimiento de sus atributos.

Los centros de atención incluidos en el estudio de caso están constituidos por el Centro de Salud Unión, las policlínicas León Duarte y Complejo Maroñas y seis consultorios de medicina familiar.

Se realizó un muestreo no probabilístico por criterios para la selección de los tres grupos de actores mencionados. Los criterios de selección consideraron el tipo de unidad asistencial: Centro de salud, policlínica y consultorios de Medicina Familiar. Por otra parte, se tomó en cuenta la producción (número de consultas) y servicios ofrecidos. En el caso de los usuarios, se asignó un número de entrevistas a cada Centro en forma proporcional a la producción de los mismos. Posteriormente, se distribuyeron las entrevistas de manera proporcional en los cinco días de la semana en que los centros están abiertos cubriendo todo el horario de atención y la cartera de servicios ofrecida. Los usuarios fueron entrevistados en la sala de espera. Se incluyó a todos los usuarios adultos y a los acompañantes de los menores de edad y/o adultos que no pudieran responder por sí mismos.

Previamente a la realización de las encuestas se realizó una experiencia piloto donde se capacitó a la utilización del instrumento a los encuestadores y se realizaron las definiciones operacionales.

En el caso de los profesionales, se entrevistaron médicos y no médicos de primer contacto de las diferentes unidades asistenciales. En el caso de los gestores, 
se entrevistaron todos los gestores del Centro de Salud y sus centros dependientes, así como la dirección regional de la RAP, la dirección de la RAP Montevideo y la dirección Nacional del Primer Nivel de Atención.

La aplicación de los instrumentos se realizó, en el caso de los profesionales y gestores, previa coordinación de la entrevista. En el caso de los usuarios, los mismos fueron entrevistados en los centros de atención seleccionados al momento de solicitar atención. La duración promedio de cada entrevista fue de 30 minutos y las mismas fueron realizadas por un grupo de 7 encuestadores, médicos residentes de Medicina Familiar y de Administración de Servicios de Salud y una estudiante avanzada de medicina. Al momento de la encuesta se solicitó a los entrevistados firmar un formulario de consentimiento informado preparado a tal efecto. Las encuestas se realizaron entre agosto y octubre de 2011.

El proceso de análisis implicó el análisis descriptivo de las variables sociodemográficas. Posteriormente, se compararon las subdimensiones dentro de los diferentes atributos de la APS para las diferentes categorías de entrevistados. Finalmente, se construyó un índice de desempeño cuyo puntaje va de 1 a 6 ponderando la frecuencia absoluta de las respuestas en la escala y el número de ítems de cada atributo.

Cada cuestionario tiene entre 80 y 88 preguntas específicas que corresponden a un indicador relacionado con una determinada dimensión de atención básica. Existían 6 opciones para cada pregunta y esas opciones fueron iguales para cada categoría de actor entrevistado (profesional, gestor o usuario) (ALMEIDA, 2006). Cada respuesta, (nunca, casi nunca, algunas veces, muchas veces, casi siempre y siempre) fue convertida a un escore o escala que varió entre 1 y 6 (siendo "1" nunca, " 2 " casi nunca, " 3 " algunas veces, etc.). En el caso de que la respuesta adecuada fuera "nunca" y la inadecuada "siempre" se invertía el escore. Luego, los escores de los indicadores fueron sumados para construir un índice compuesto para cada dimensión. Por ejemplo, todos los escores de las preguntas sobre acceso fueron sumados y la media de esa suma representó el índice compuesto acceso (ALMEIDA, 2006). Este mismo proceso fue seguido para construir un índice compuesto para cada dimensión en la cual la escala de puntaje otorgado va de desempeño 1 (peor calificado) a desempeño 6 (mejor calificado).

\section{Resultados}

Se realizaron un total de 178 entrevistas a usuarios, 25 entrevistas a profesionales y 5 entrevistas a gestores. Del total de usuarios, correspondieron el $82 \%$ a usuarias de sexo femenino con una media de edad de 53,8 ańos para ambos sexos.

\section{Atributos de APS}

En relación a la calificación de desempeño de los atributos de APS desde la perspectiva de los usuarios (ver Gráfico 1) y teniendo en cuenta que el máximo puntaje es 6 , la formación profesional fue el atributo mejor calificado obteniendo un puntaje de 5,5, seguido de vínculo (con un puntaje de 5,3), cartera de servicios $(5,1)$, puerta de entrada ( 5,0 puntos) y coordinación (4,7 puntos).

En el otro extremo, los atributos que obtuvieron la peor calificación fueron enfoque familiar (3,6 puntos), acceso (3,4 puntos) y orientación a la comunidad (3,3 puntos).

Desde la perspectiva de los profesionales, la puerta de entrada fue el atributo mejor calificado obteniendo un puntaje de 5,5 (ver Gráfico 1). Le siguen cartera de servicios (con un puntaje de 4,9), formación profesional $(4,8)$ y coordinación y vínculo (ambos con 4,6 puntos). En el otro extremo, los atributos que obtuvieron la peor calificación fueron orientación a la comunidad (3,6 puntos) y acceso (3,3 puntos).

Por último, desde la perspectiva de los gestores la cartera de servicios fue el atributo mejor calificado obteniendo un puntaje de 5,4 (ver Gráfico 1). Le siguen la puerta de entrada (con un puntaje de 5,2), la coordinación $(4,8 \%)$ y la formación profesional (4,7 puntos). En el otro extremo, los atributos que obtuvieron la peor calificación fueron el acceso y la orientación a la comunidad con 3,6 y 3,7 puntos respectivamente. 
Gráfico 1. Evaluación del desempeño de los atributos de APS desde la perspectiva de los usuarios, profesionales y gestores, C. de Salud Unión, 2011

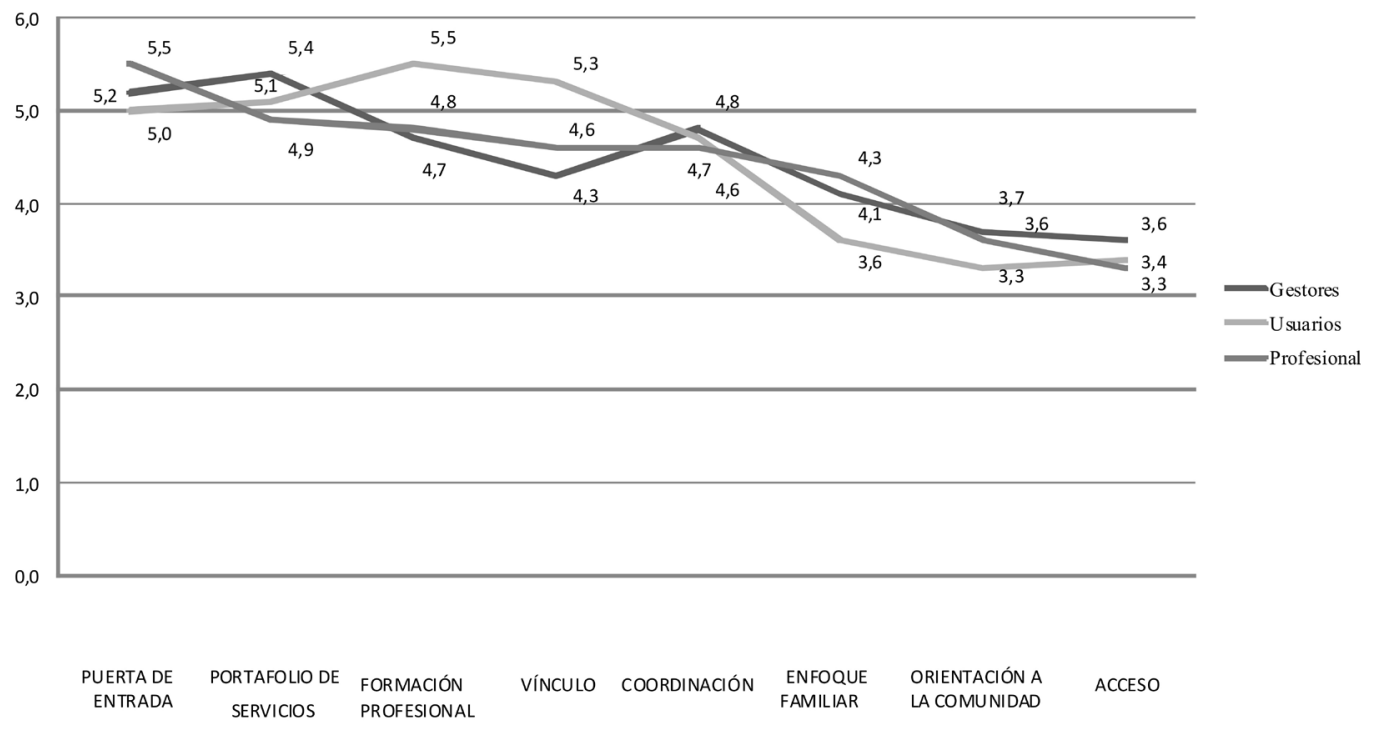

Desde una perspectiva global, tanto los profesionales como los usuarios y los gestores calificaron globalmente los atributos con un puntaje de 4,5.

Asimismo, teniendo en cuenta a los tres grupos de actores a la vez, el orden de atributos con puntaje decreciente (el mejor calificado en fila superior y el peor calificado en fila inferior), sería (Gráfico 1):

1. Puerta de entrada

2. Portafolio de servicios

3. Formación profesional

4. Vínculo

5. Coordinación

6. Enfoque familiar

7. Orientación a la comunidad

8. Acceso

Sin embargo, mientras que para los gestores el ítem mejor calificado es la carteara de servicios (5,4 puntos), para los profesionales lo es la puerta de entrada $(5,5$ puntos) y para los usuarios la formación profesional
(5,5 puntos). En general, existe coincidencia en que los ítems peor calificados son enfoque familiar, orientación a la comunidad y acceso, aunque gestores y profesionales coinciden en que el acceso es el atributo con peor calificación, mientras que para los usuarios es la orientación comunitaria.

Los usuarios poseen una perspectiva más favorable para los atributos formación profesional y vínculo en relación a gestores y profesionales, sin embargo poseen una perspectiva menos favorable en el caso de orientación comunitaria, enfoque familiar y, en menor magnitud, en puerta de entrada.

\section{Acceso}

Con respecto a la accesibilidad, si bien el puntaje global fue bajo en relación al resto de los atributos, (3,3 puntos para usuarios y profesionales y 3,6 para gestores) obtuvo una excelente calificación la accesibilidad económica (6,0 puntos para usuarios y gestores y 5,9 puntos para profesionales).

En este sentido, la inaccesibilidad estaría vinculada básicamente a aspectos organizacionales y funcionales según la perspectiva de los tres actores, aunque existen diferencias en la percepción de determinados 
componentes del atributo según el actor considerado (ver Gráfico 2).

En el caso de los usuarios, valoran de manera más positiva la oferta de medicamentos y la facilidad para obtener una consulta médica en el centro en relación a los profesionales y gestores. Sin embargo, el tiempo de espera mayor a 30 minutos para ser atendido tiene para los usuarios un puntaje muy bajo en relación a los otros actores considerados (ver Gráfico 2). La perspectiva de oferta de medicamentos esenciales adecuada contrasta sin embargo con la valoración realizada por los profesionales, quienes otorgaron el puntaje más bajo (ver Gráfico 2). Asimismo, la perspectiva de los gestores es más favorable en relación a los tiempos de espera, la disponibilidad de teléfono para citas y la atención de pacientes en los fines de semana (ver Gráfico 2).

\section{Puerta de entrada}

En cuanto al atributo puerta de entrada, (ver Gráfico 1) la calificación global obtuvo un puntaje elevado (5,0 puntos para usuarios, 5,5 para profesionales y 5,2 puntos para gestores). Este atributo se encuentra ubicado en el primer lugar por parte de los profesionales y en el segundo lugar por parte de los gestores.

Los usuarios calificaron también con muy buen puntaje todas las subdimensiones que componen el atributo (utilización del servicio para actividades preventivas: 5,1 puntos, requerimiento de consulta con un generalista antes de acceder a una consulta con especialista: 5,0 puntos y utilización del servicio ante problemas de salud: 4,8 puntos).

Gráfico 2. Desempeño de los componentes del atributo acceso según usuarios, gestores y profesionales, C. de Salud Unión, 2011

\section{ACCESO}

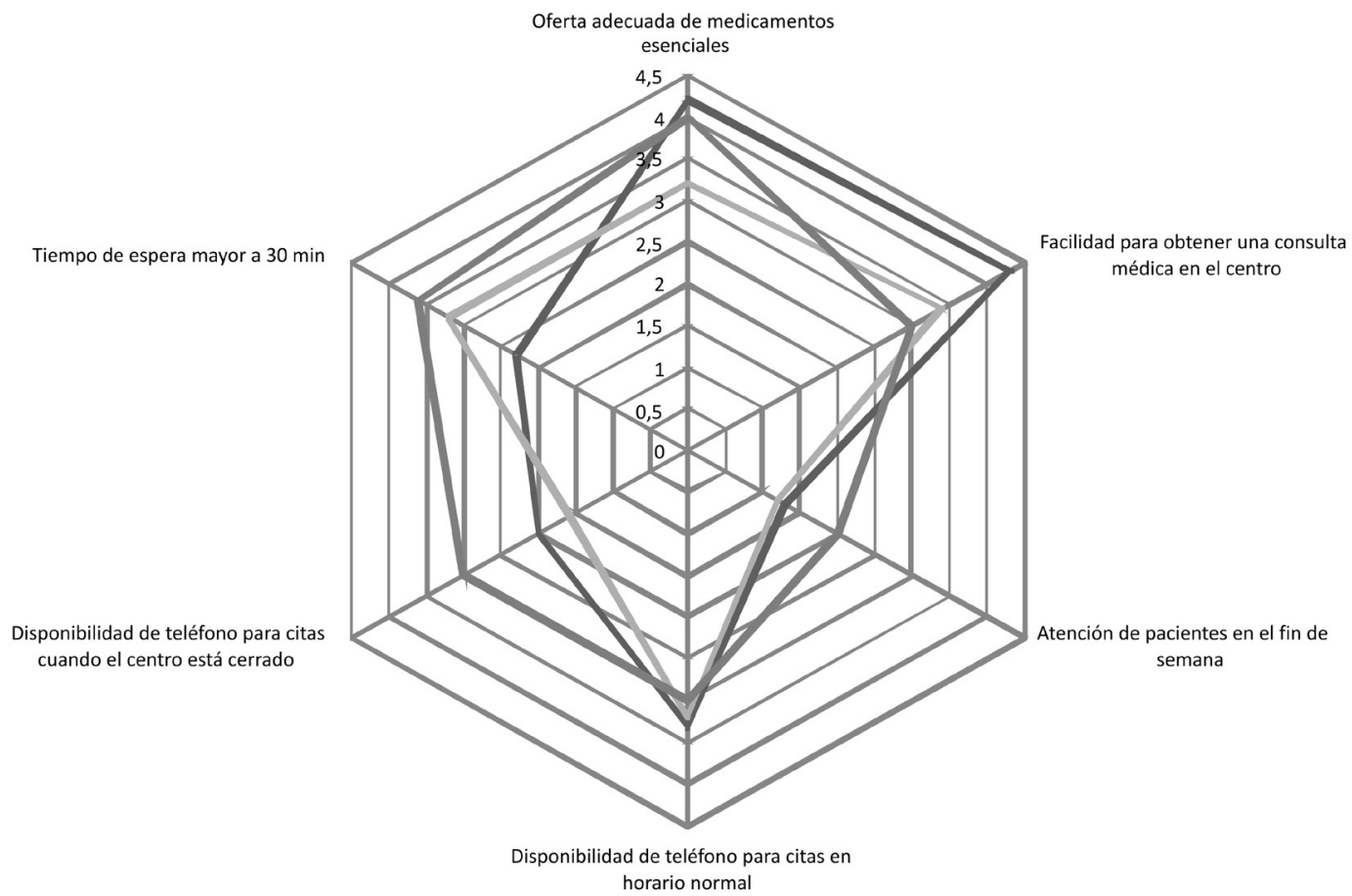




\section{Vínculo}

En relación al vínculo, en general se destaca que los usuarios calificaron con mejor puntaje que el resto de los actores (profesionales y gestores) prácticamente todas las dimensiones (Gráfico 3). El elevado puntaje otorgado por los usuarios para el atributo globalmente (5,3 puntos) lo colocó como el atributo que ocupa el segundo lugar.

Para los profesionales obtuvo un puntaje global de 4,6 . En general todas las subdimensiones son calificadas con un muy buen puntaje para ésta categoría de actores y se destacan la posibilidad de los usuarios de acceder al mismo profesional que lo atiende en el caso de tener una duda sobre su tratamiento $(5,4$ puntos) y la utilización por parte de los profesionales de la misma historia clínica en cada consulta del usuario con un puntaje de 5, 3 (Gráfico 3).
La calificación del vínculo por parte de los gestores obtuvo un puntaje global de 4,3. Las subdimensiones calificadas con mayor puntaje son la posibilidad de los usuarios a acceder al mismo profesional en el caso de tener una duda sobre su tratamiento $(5,4$ puntos) y la utilización por parte de los profesionales de la misma historia clínica en cada consulta del usuario con un puntaje de 4,8. Sin embargo, las subdimensiones calificadas con menor puntaje son las que exploran si los profesionales son informados cuando el usuario no consigue obtener un medicamento prescrito ( 3,8 puntos) y la adscripción de la población usuaria al Centro (3,5 puntos). Se destaca por otro lado que, en general, los gestores son quienes poseen una percepción más desfavorable del atributo en prácticamente todos sus componentes. (Gráfico 3)

Gráfico 3. Desempeño de los componentes del atributo vínculo según usuarios, gestores y profesionales, C. de Salud Unión, 2011

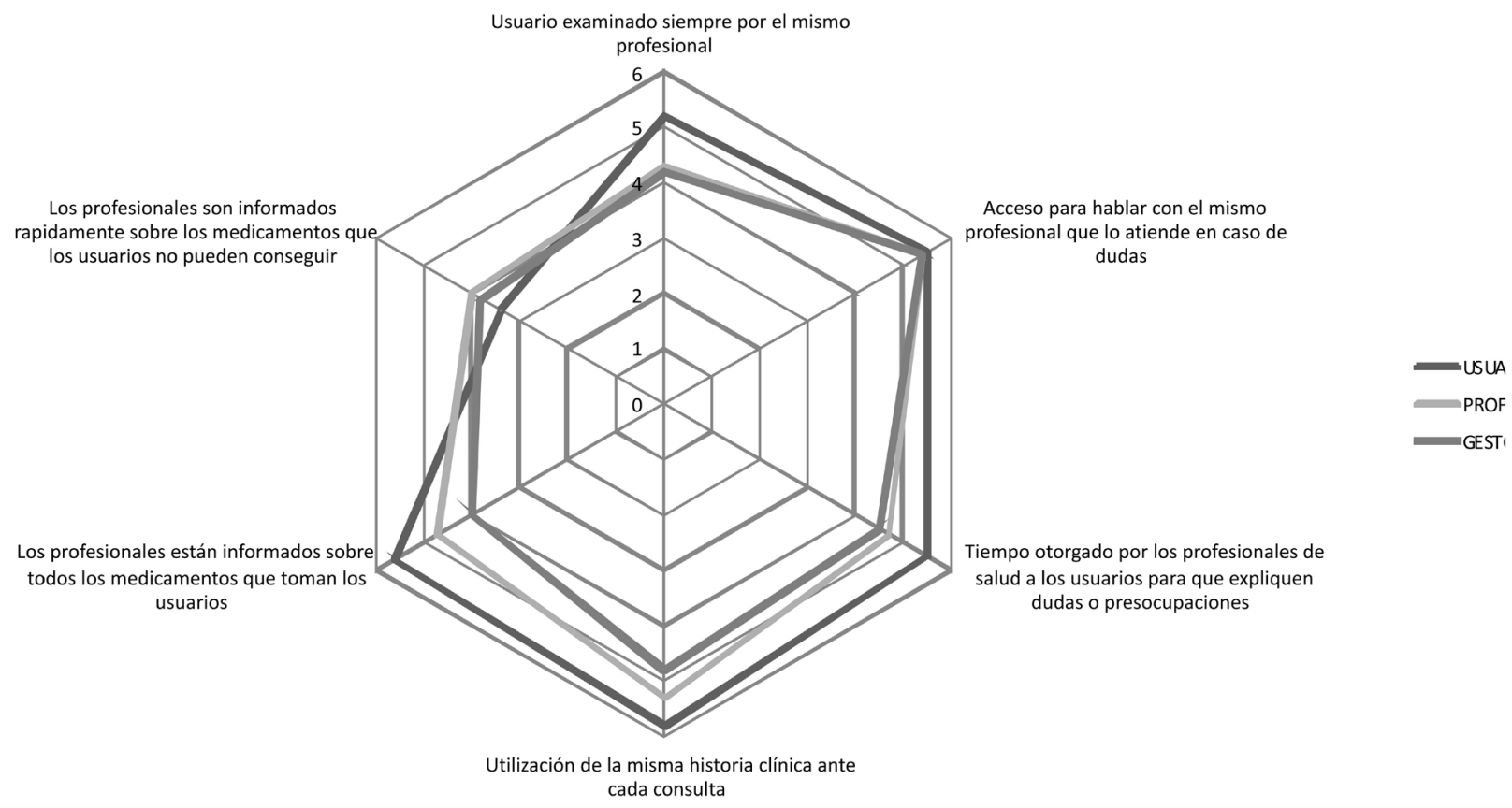




\section{Cartera de servicios}

La cartera de servicios, según la perspectiva de los usuarios, obtuvo un excelente puntaje (5,1 puntos) (Gráfico 1) con una percepción global de buena disponibilidad de servicios en el Centro, ya sean preventivos o terapéuticos. Dentro de éstos, los menores puntajes fueron obtenidos para las prestaciones educativas y de consejería.

Para los profesionales, obtuvo el segundo mejor puntaje dentro de los atributos de la APS (4,9 puntos) y en ningún caso las prestaciones exploradas bajan de 4 puntos excepto el control y tratamiento de la tuberculosis $(3,5$ puntos) y la realización de cirugías ambulatorias (1,1 puntos).

Según los gestores, la cartera de servicios obtuvo el mejor puntaje dentro de los atributos de la APS (5,4 puntos). De las 21 subdimensiones exploradas, se destacan siete con un puntaje excelente (6 puntos), 12 con puntaje casi excelente (entre 5,8 y 5,4 puntos) y dos mal calificadas (control y tratamiento de la TBC (3,4 puntos) y las cirugías ambulatorias (1,2 puntos)).

Desde una perspectiva comparada, se destaca que, en general, en todos los servicios la percepción de los gestores es francamente más favorable que la de los usuarios y profesionales, existiendo varias prestaciones a las cuales los gestores otorgaron puntajes máximos.

\section{Coordinación}

El atributo coordinación obtuvo un índice global de 4,7, ocupando el 4to lugar desde la perspectiva de los usuarios y de los profesionales y el tercer lugar para los usuarios. Desde una perspectiva comparada de actores, la calificación del atributo coordinación fue buena (ver Gráfico 4), existiendo solo un componente con una calificación muy baja por parte de profesionales $(2,1$ puntos) y gestores (1,6 puntos) (cita automática para conocer los resultados de los exámenes), lo que contrasta con el puntaje otorgado por los usuarios (4,1 puntos).

Gráfico 4. Desempeño de los componentes del atributo coordinación según usuarios, gestores y profesionales, C. de Salud Unión, 2011

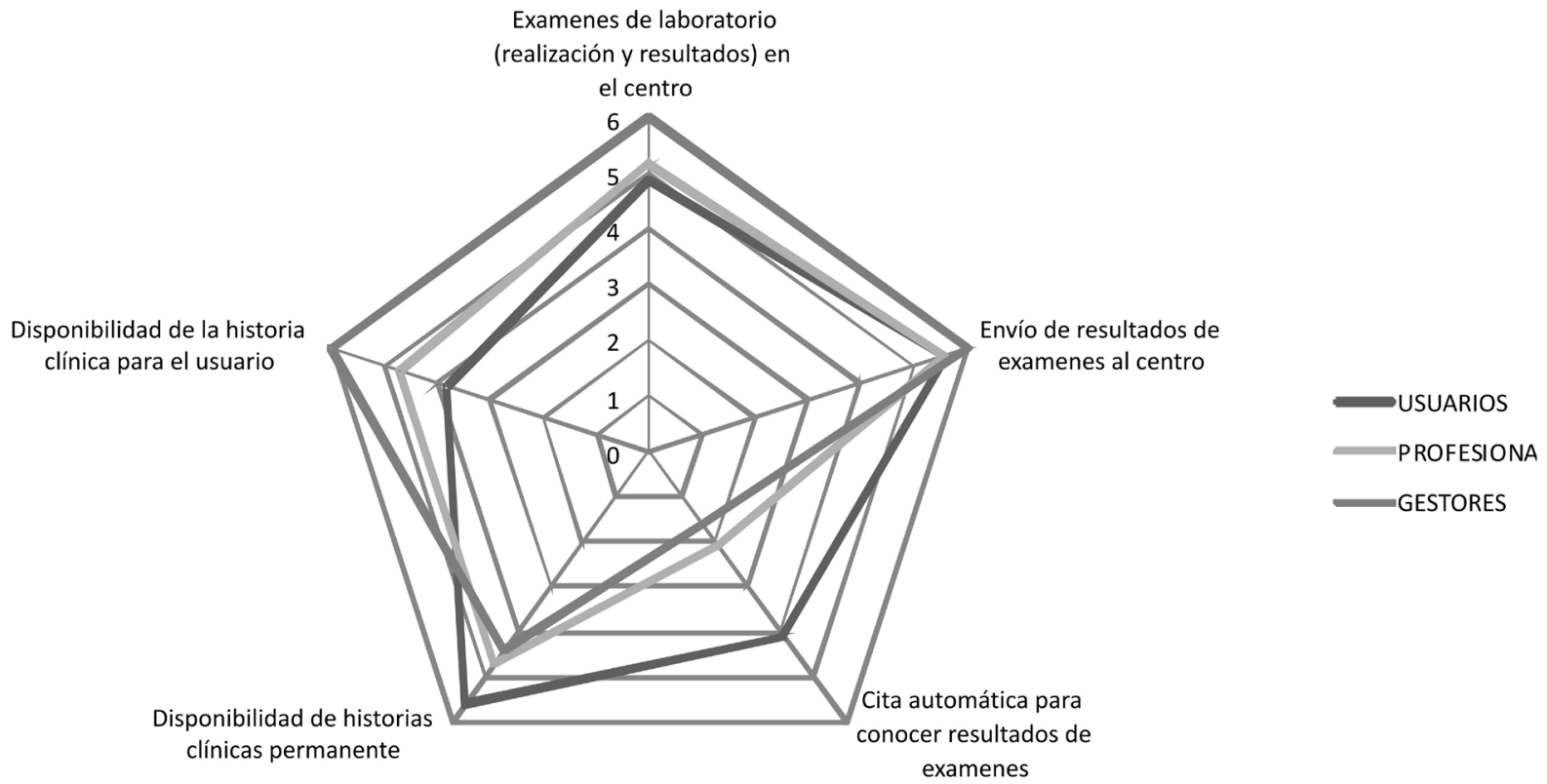


Las tres subdimensiones con puntaje más bajo para los usuarios fueron: la posibilidad de conocer la historia clínica (3,8 puntos), el acuerdo del lugar de derivación para interconsultas con especialistas (3,8 puntos) y la ayuda para obtener la cita para la interconsulta $(3,5$ puntos). En el caso de los profesionales, las subdimensiones con puntaje más bajo fueron: la supervisión periódica para revisar las necesidades de referencia a otros niveles de atención y la existencia de auditoría periódica de historias clínicas con un puntaje de 2, 7; en ambos casos, la consulta al usuario sobre la disponibilidad de tiempo para otorgar una cita de control (2,6 puntos) y la posibilidad de acceder automáticamente a una consulta de control para conocer el resultado de exámenes (2,1 puntos). Para los gestores, los atributos peor calificados fueron: si los usuarios son consultados sobre su disponibilidad de tiempo para una cita de control con 3, 8 puntos, si los profesionales de APS reciben información escrita sobre los resultados de las consultas referidas al especialista y si existe auditoría periódica de las historias clínicas o los registros médicos, en los dos casos calificados con 2,2 puntos. Por último, la subdimensión peor calificada ( 1,6 puntos) fue si la cita para la consulta de control del usuario para conocer los resultados de los exámenes es realizada automáticamente por el Centro de Atención. En cuanto a la disponibilidad de la historia clínica para el usuario, los gestores otorgaron un puntaje máximo, los profesionales un puntaje menor ( 4,7 puntos) y los usuarios otorgaron el puntaje más bajo (3,8 puntos).

\section{Enfoque familiar}

Si bien la calificación del enfoque familiar fue baja en las tres categorías de actores (3,6 puntos para los usuarios, 4,3 puntos para los profesionales y 4,1 puntos para los gestores), para los usuarios fue particularmente más baja en dos subcomponentes del atributo: indagación de Antecedentes Familiares en la consulta e indagación sobre las condiciones de vida de los pacientes (desempleo, disponibilidad de agua potable, saneamiento, etc.)

Los gestores otorgaron una puntuación particularmente baja a la orientación familiar de la Historia clínica y para los profesionales, el ítem peor calificado es el relativo a qué porcentaje de historias clínicas son realizadas con enfoque familiar (3,2 puntos).

\section{Orientación a la comunidad}

Para los usuarios, la orientación a la comunidad fue el atributo que recibió la peor calificación (3,3 puntos). El ítem con peor calificación es el referido a si el usuario o la familia son consultados para saber si los servicios del Centro atienden sus problemas de salud (2,3 puntos). (Gráfico 5)

Gráfico 4. Desempeño de los componentes del atributo coordinación según usuarios, gestores y profesionales, C. de Salud Unión, 2011

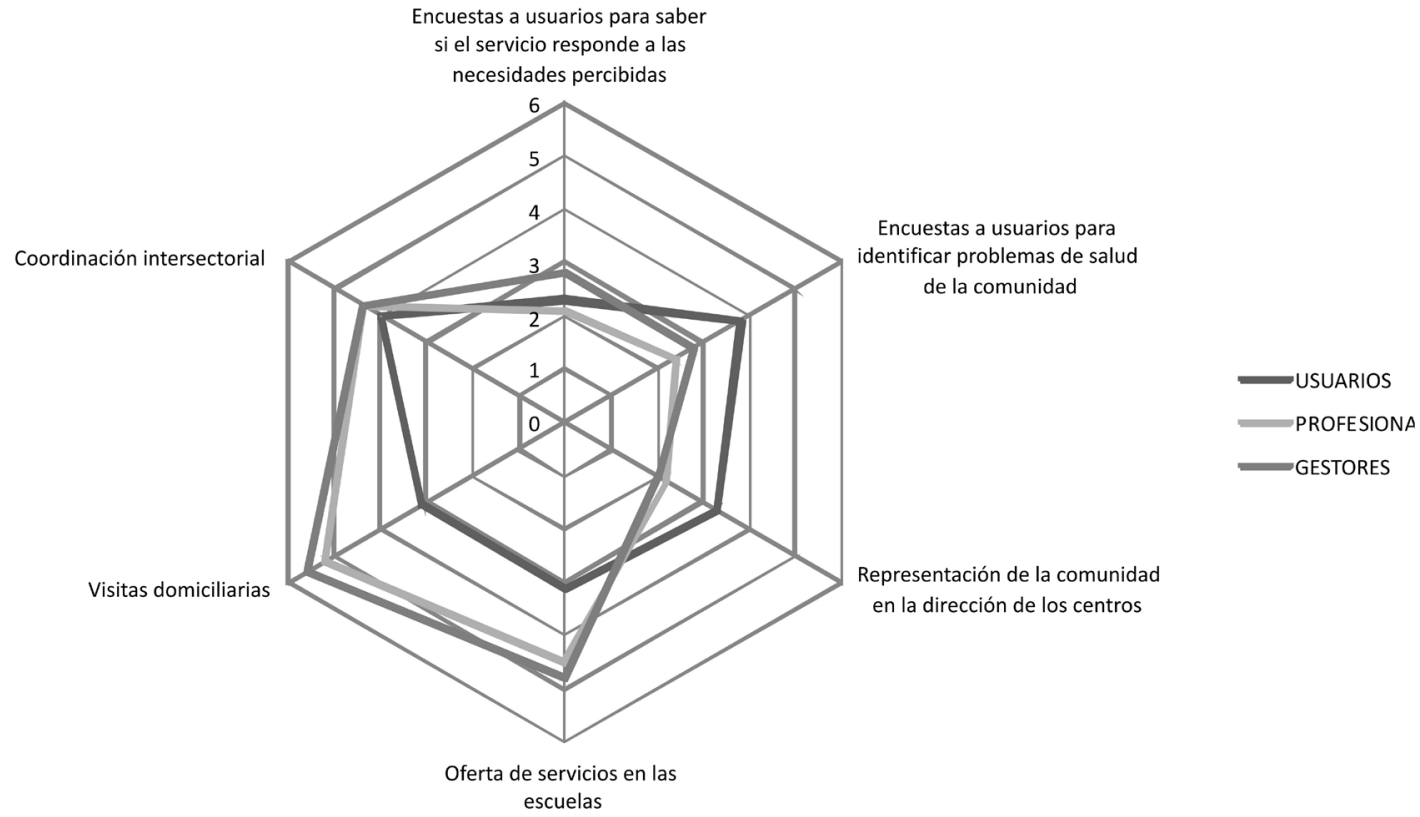


Para los profesionales, el puntaje global otorgado fue de 3,6, siendo el segundo puntaje más bajo luego del acceso. Por último, este atributo fue calificado por los gestores con un puntaje global de 3,7, siendo el segundo puntaje más bajo luego del acceso. (Gráfico 5).

Desde una perspectiva comparada entre los actores, si bien la calificación global fue baja, existe una visión más negativa en los usuarios para los subcomponentes coordinación intersectorial, visitas domiciliarias y oferta de servicios en las escuelas, mientras que la perspectiva de los usuarios es más favorable en relación a la representación de la comunidad en la dirección de los centros y encuestas a usuarios para identificar problemas de salud de la comunidad(Gráfico 5).

\section{Formación profesional}

El atributo formación profesional fue el mejor calificado en lo global desde la perspectiva de los usuarios $(5,5$ puntos) y también en cada ítem particular, recibiendo siempre puntajes por encima de 5 (disponibilidad permanente de profesionales para la atención, recomendación del Centro a una amigo, resolutividad de los problemas de salud y relacionamiento con la comunidad).

Para los profesionales obtuvo un puntaje medio de 4,8, pero en ningún caso las subdimensiones se valoraron con menos de 3,9 puntos. Por último, para los gestores, la formación profesional obtuvo un puntaje medio de 4,7.

\section{Discusión y Conclusiones}

Uruguay transita desde el año 2005 un proceso de reforma sanitaria con la creación de un Sistema Nacional Integrado de Salud (SNIS) que favorece el desarrollo de la APS desde una concepción integradora (OLESKER; GONZÁLEZ, 2009). Según los resultados del análisis panorámico realizado en la primera fase de la investigación, se identifican acciones a nivel macrosocial que apuntan a superar la segmentación del sistema y la fragmentación de los cuidados. En este sentido, se hacía mención al reciente desarrollo de un marco normativo con cambios que favorecen el desarrollo de la APS (entre otras, Ley del Sistema Nacional Integrado de Salud).
Sin embargo, se constataba que estas acciones no se habían traducido necesariamente en cambios a nivel operativo (SOLLAZZO; BERTERRETCHE, 2011).

El estudio de caso se realizó al interior de los servicios públicos de ASSE. Como lo explicita su misión, ASSE participa del contexto de reforma tomando como eje central el desarrollo de la estrategia de APS. El estudio de caso aborda el desempeño de la APS en un nivel operativo, el Centro de Salud Unión y sus policlínicas dependientes ubicados en el área urbana del Departamento de Montevideo.

Desde un punto de vista general, se observa una buena calificación de desempeńo a nivel global en los atributos: puerta de entrada, cartera de servicios y formación profesional. Los atributos de nivel intermedio de desempeño corresponden a vínculo y coordinación. En el otro extremo, los atributos con menor nivel de desempeño fueron el enfoque familiar y comunitario y el acceso. En este sentido, las barreras al acceso están vinculadas básicamente a aspectos organizacionales y funcionales de forma coincidente en los tres grupos de actores entrevistados.

A pesar de la visión concordante entre las 3 categorías de actores, existen algunas diferencias específicas entre las que destacamos que los atributos vínculo y coordinación recibieron menores puntajes en la calificación entre gestores y profesionales, no tanto así en los usuarios.

Por otra parte, si bien el acceso y la orientación familiar y comunitaria recibieron el puntaje más bajo, para los gestores, el atributo con menor nivel de desempeńo fue el acceso, mientras que para profesionales y usuarios fue la orientación comunitaria.

Los resultados del estudio de caso son consistentes con los resultados de la primera fase del estudio multicéntrico. En este sentido, existe un desempeño medio de los atributos vínculo y coordinación con dificultades evidentes en el acceso que hacen a la fragmentación de los cuidados. La coordinación aparece como un factor clave en el fortalecimiento de la APS (ALMEIDA, 2011).

La baja calificación de la orientación familiar y comunitaria de los profesionales de la salud también 
nos refiere a las dificultades para responder a la estrategia de APS por parte de los profesionales que se describía en la primera fase. En este sentido, se plantea que los sistemas de salud basados en APS imponen grandes desafíos para los sistemas de recursos humanos (MACINKO, 2007).
Si bien la estrategia de APS está consagrada a nivel macro social a través de un marco normativo expresado en la Ley de creación del SNIS, su implementación a nivel operativo implica importantes desafíos para el logro de una adecuada integración y coordinación de los cuidados.

\section{Referências}

ALMEIDA, P.F.; FAUSTO, M.C.R; GIOVANELLA, L. Fortalecimento da atenção primária à saúde: estratégia para potencializar a coordenação dos cuidados. Revista Panamericana de Salud Publica, v. 29, n. 2, p. 84-95, 2011

ADMINISTRACÍON DE LOS SERVIÇOS DE SALUD DEL ESTADO (ASSE). Documento de trabajo de la Dirección de la Administración de Servicios de Salud del Estado. 2011. Disponível em: <http://www. asse.com.uy/uc_5171_1.html>. Acesso em: 7 fev 2012.

Objetivos Institucionales. 2010. Disponível em: <http:// www.asse.com.uy/uc_2113_1.html>. Acesso em: 10 fev 2012.

BENIA, W et al. ASSE en la Reforma de la Salud. In: Transformar el futuro: Metas cumplidas y desafíos renovados para el Sistema Nacional Integrado de Salud. Montevideo: Ministerio de Salud Pública, 2011.

CASSADY, C. H. et al. Measuring Consumer Experiences With Primary Care. Pediatrics, v. 105, n.4, abr. 2000.

CRUZ, M. Descripción del Centro de Salud Unión. Documento de trabajo. Montevideo: RAP - ASSE, 2011. Mimeo.

LEVCOVITZ, E, et al. (ed.) Atención Primaria de Salud en Uruguay: experiencias y lecciones aprendidas. Montevideo: OPS; 2011

MACINKO, J. et al. La renovación de la atención primaria de salud en las Américas. Rev Panam Salud Publica, v. 21, n. 2-3, p. 73-84, 2007.
OLESKER, D.; GONZÁLEZ, T. La construcción del Sistema Nacional Integrado de Salud (2005-2009). Montevideo: Ministerio de Salud Pública; 2009.

ORGANIZACIÓN PANAMERICANA DE LA SALUD (OPS). La Renovación de la Atención Primaria de Salud en las Américas. 2007. Disponível em: <http://www.paho.org/spanish/AD/THS/OS/APS_ spa.pdf>. Acesso em: 25 mai 2012.

SOLLAZZO, A.; BERTERRETCHE, R. El Sistema Nacional Integrado de Salud en Uruguay y los desafíos para la Atención Primaria. Ciência \& Saúde Coletiva, v. 16, n. 6, p. 2829-2840, 2011.

VEGA ROMERO, R.; MARTÍNEZ COLLANTES, J.; ACOSTA RAMIREZ, N. Evaluación rápida del desempeño de la red pública de servicios de salud de Suba en el logro de los atributos de la Atención Primaria de Salud - APS1. Revista Gerencia y Políticas de Salud, v.8, n.16, p. 165-190, 2009. Disponible en: <http://www.scielo.org.co/scielo. php?script=sci_arttext\&pid=S1657-70272009000100010\&Ing=es \&nrm=iso>. Acesso em: 06 mar. 2012

Recebido para publicação em Abril/2012

Versão definitiva em Agosto/2012

Suporte finaceiro: International Development Research Centre (IDRC)

Conflito de interesse: inexistente 